\section{GDF11: a fountain of youth for the ageing COPD lung?}

\author{
Ma'en Obeidat, ${ }^{1}$ Don D $\operatorname{Sin}^{1,2}$
}

Smoking is the single most important causal risk factor for COPD. The public health campaigns and policies have worked to reduce smoking rates by over $50 \%$ since those of the 1970 s and are now at an all-time low in the $\mathrm{UK},{ }^{1}$ Canada and throughout most of the western world. Yet, curiously and paradoxically, the burden of COPD (as measured by its prevalence, hospitalisation and mortality rate) is at an all-time high in these countries, and over the next 20 years, the burden of COPD is expected to more than double. ${ }^{2}$ Why?

The answer is a simple math issue related to ageing. Most industrialised countries of the world are getting older and, unquestionably, COPD is an age-related disorder. Whereas COPD is almost unheard of in individuals less than 40 years of age (even among heavy smokers), 1 in 10 lifetime never-smokers and 1 in 3 develop COPD by age $75 .^{3}$ This notion of accelerated ageing of COPD is supported by animal models, which demonstrate premature appearance of emphysematous lungs in mice, which have genetically altered age-related pathways (eg, Klotho mice). ${ }^{4}$

Physiologically, ageing can be defined as the progressive decline in homeostasis and loss of tissue and organ functions over time. ${ }^{5}$ Ageing is a risk factor for many (non-communicable) chronic conditions beyond COPD including cancer, congestive heart failure, dementia, diabetes and osteoarthritis. ${ }^{5}$ The aetiology of ageing is unknown, but the most popular theory involves accumulation of reactive oxygen species (ROS) related to excess oxidative stress. ${ }^{6}$ At a cellular level, ageing manifests as an irreversible loss of proliferative capacity of viable mitotic cells, termed cellular senescence. Unlike apoptotic cells, senescent cells remain metabolically active and display a senescence-associated

\footnotetext{
${ }^{1}$ The University of British Columbia Centre for Heart Lung Innovation, St Paul's Hospital, Vancouver, British Columbia, Canada

${ }^{2}$ Respiratory Division, Department of Medicine, University of British Columbia, Vancouver, British Columbia, Canada
}

Correspondence to Dr Don D Sin, Respiratory Division, Department of Medicine, University of British Columbia, Vancouver V6Z 1Y6, British Columbia, Canada; don.sin@hli.ubc.ca secretory phenotype characterised by the production of proinflammatory cytokines such as interleukin (IL)-6, IL-8 and matrix metalloproteinases (MMPs). ${ }^{7}$ Despite the importance of the ageing process in COPD, the exact molecular drivers of the senescent lung of COPD remain largely a mystery.

One very interesting age-related protein that may modulate senescence is growth differentiation factor (GDF) 11. GDF11 is a member of the transforming growth factor beta family of proteins. In 2014, GDF11 was named a runner-up for Science magazine's breakthrough of the year. ${ }^{8}$ In a striking study of heterochronic parabiosis in which two mice of different ages were surgically joined to share the same circulation, GDF11 was identified as a circulating factor that reversed cardiac hypertrophy. ${ }^{9}$ This observation was advanced by a study in humans, which demonstrated a possible cardioprotective role of GDF $11{ }^{10}$ Interestingly, in this study, GDF11 was associated with reduced total (and not just cardiovascular) mortality suggesting broader benefits beyond the cardiovascular system. The finding was particularly notable given the fact that GDF11 is widely expressed in a variety of different human tissues and organs. ${ }^{10}$ Intriguingly, other studies in mice demonstrated the benefits of GDF11 supplementation in reversing age-related dysfunction in skeletal muscle, ${ }^{11}$ improving cerebral blood flow and promoting neurogenesis in the brain. ${ }^{12}$ The effects of GDF11 in COPD, however, were unknown.

Onodera et al conducted a series of translational studies to delineate the possible role of GDF11 in the pathogenesis of COPD. ${ }^{13}$ First, the authors found that plasma GDF11 levels were significantly related to lung function, as measured by $\mathrm{FEV}_{1} \%$ predicted and were significantly decreased in patients with COPD compared with control never-smokers or ex-smokers. Importantly, these relationships were replicated in an independent cohort. Interestingly, GDF11 levels did not correlate with age of the patients. This may be due to the fact that GDF11 is a marker of biological ageing and cellular senescence rather than that of chronological ageing. An alternate explanation is that the two cohorts of this study had a relatively narrow range of age, which may have obscured the effects of age on GDF11 levels.

Moving on to in vitro studies, Onodera et al used immunohistochemistry and detected GDF11 expression mainly in airway epithelial cells and airway mesenchymal cells. The protein expression in lung fibroblasts and bronchial epithelial cells of subjects with COPD were significantly reduced compared with those of controls. Importantly, protein markers of senescence, namely p53 and p16, were significantly increased in lung fibroblasts from patients with COPD, relative to those from controls, and the expression levels were inversely related to GDF11 expression.

Two main phenotypes were described in the literature for cellular senescence: replicative, characterised by telomere shortening, and premature senescence, which can be stress induced. ${ }^{67}$ To identify which of these processes are affected by GDF11 in the context of COPD, human lung fibroblasts (HFL-1) were exposed to cigarette smoke extract (CSE), which led to decreased GDF11 production, as well as increased expression of p21, p53 and the senescence-associated $\beta$-galactosidase (SA- $\beta$-gal). GDF11 production was rescued when cells were pretreated with the antioxidant $\mathrm{N}$-acetylcysteine. Treatment of fibroblasts and primary bronchial epithelial cells with GDF11 also inhibited the CSE-induced SA- $\beta$-gal production and cell proliferation. However, replicative senescence, defined as HFL-1 cells cultured to the 27th passage, was not affected by GDF11 treatment. However, the authors did not measure telomere length in these cells to confirm replicative senescence. Although preliminary, the fact that GDF11 levels were not significantly related to age and GDF11 treatment did not affect replicative senescence in vitro suggests its effects are specific to stress-induced or premature senescence.

Next, the authors determined the role of GDF11 on oxidative stress. They found that GDF11 mitigated the CSE-induced ROS activity, suppressed the release of IL-8 and MMP-9, and restored tissue repair in fibroblasts. Their subsequent finding that GDF11 phosphorylated Smad2/3 offered some insights into the mechanisms of GDF11 signalling. Of greater relevance to COPD, the authors used primary cells from patients with COPD and found that GDF11 suppressed the expression of senescence markers p16 and SA- $\beta$-gal in these COPD lung cells. Although these findings could have been confounded by selective expansion of 
non-senescent population of cells, these experiments, nevertheless, raise the possibility that GDF11 may have therapeutic potential to treat or even reverse cellular senescence in COPD.

The authors then went on to evaluate the effect of GDF11 on emphysema using an in vivo system. They used an elastase mouse model of emphysema treated with recombinant GDF11 for 21 days. While elastase exposure reduced the production of GDF11 and augmented p16 and p53 production, GDF11 treatment protected the mice against cellular senescence and mitigated the development of emphysema related to elastase instillation. It should be noted, however, the elastase model induces acute lung injury and may not adequately mimic clinical COPD, which is characterised by chronic inflammation and aberrant repair. Nevertheless, taken together, this series of experiments suggest that GDF11 is involved in the pathogenesis of COPD by protecting cells against cellular senescence and by mitigating oxidative stress, and importantly, circulating levels of GDF11 in plasma may act as a predictive biomarker for those who might benefit from GDF11 supplementation therapy.

These exciting findings by Onodera et al also provoke many questions. For instance, is senescence always detrimental and requires 'abolition'? Are there downsides to decelerating senescence such as carcinogenesis? The answer is not entirely clear. Senescence has been shown to be useful and may in fact have evolved as a mechanism to fight tumourigenesis. ${ }^{14}$ As most things in biology, too little or too much of a 'good thing' may be detrimental. Onodera et al did not fully evaluate a possible dose-response relationship between GDF11 and various readouts in their experiments. Furthermore, while these early findings suggest strongly an important role of GDF11 in COPD (at least in mice), causality in humans remains elusive.

To this end, human genetics could assist in elucidating causality by providing unidirectional flow of information from DNA to gene/protein expression and ultimately to disease. In published genome-wide association studies, GDF11 has not been significantly associated with COPD or lung function ${ }^{15}$ (accessed 20 April 2017). It may be that its effects are subtle and thus may not be able to achieve the stringent genome-wide significance. Although circulating GDF11 levels were reported to be heritable (genetically determined) in mice, ${ }^{16}$ in different human tissues from the Genotype-Tissue Expression (GTEx) project, the expression of GDF11 mRNA was not genetically determined (GTEx portal accessed 20 April 2007). Further research using genetic manipulations and gene editing in specific cells and tissues may be crucial in establishing a causal role of GDF11 in human COPD.

The findings from Onodera et al are exciting and novel and may represent a new therapeutical and biomarker target to modulate COPD and establish cellular senescence as an important player in the pathogenesis of COPD. However, before accepting GDF11 as the 'fountain of youth' in preventing or perhaps even reversing the aged lungs of patients with COPD, additional translational studies are required to address some critical mechanistic questions that will elucidate the exact role this molecule plays in the pathogenesis of COPD. These may include experiments with transgenic mice expressing GDF11 selectively in lung tissue to determine whether these mice are protected against development of air pollution or cigarette smoke-related emphysema (and whether the reverse is also true) or treatment of these animals with selective inhibitors or agonists of GDF11 for disease modulation, and most importantly proof-ofconcept studies in patients with COPD to determine whether small molecule or antibody-based treatments related to GDF11 or its pathway can ameliorate key readouts in COPD including symptoms and lung function.

Contributors DDS and MO contributed equally to the write-up of this editorial.

Competing interests DDS has received honoraria for speaking engagements from AstraZeneca, Novartis and Boehringer Ingelheim; has received stipends for serving on advisory boards of AstraZeneca, Regeneron, Sanofi and Boehringer Ingelheim; and received research funding from AstraZeneca, Merck and Boehringer Ingelheim. MO has no competing interests to declare.

Provenance and peer review Commissioned; externally peer reviewed.

(c) Article author(s) (or their employer(s) unless otherwise stated in the text of the article) 2017. All rights reserved. No commercial use is permitted unless otherwise expressly granted.

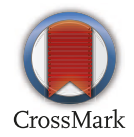

To cite Obeidat M'en, Sin DD. Thorax 2017;72:874-875.
Published Online First 11 August 2017

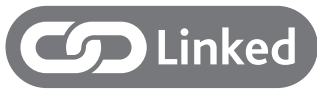

http://dx.doi.org/10.1136/thoraxjnl-2016-209352

Thorax 2017:72:874-875.

doi:10.1136/thoraxjnl-2017-210359

\section{REFERENCES}

1 The Guardian. Number of smokers in England drops to all-time low: Secondary Number of smokers in England drops to all-time low 2016. https://www.theguardian. com/society/2016/sep/20/number-of-uk-smokers-fallsto-lowest-level (accesed 20 Sep 2016)

2 Khakban A, Sin DD, FitzGerald JM, et al. The projected epidemic of chronic obstructive pulmonary disease hospitalizations over the next 15 years. A populationbased perspective. Am J Respir Crit Care Med 2017;195:287-91.

3 Pelkonen M, Notkola IL, Nissinen A, et al. Thirty-year cumulative incidence of chronic bronchitis and COPD in relation to 30-year pulmonary function and 40-year mortality: a follow-up in middle-aged rural men. Chest 2006:130:1129-37.

4 Fukuchi Y. The aging lung and chronic obstructive pulmonary disease: similarity and difference. Proc Am Thorac Soc 2009;6:570-2.

5 Childs BG, Durik M, Baker DJ, et al. Cellular senescence in aging and age-related disease: from mechanisms to therapy. Nat Med 2015;21:1424-35.

6 Barnes PJ. Senescence in COPD and Its Comorbidities. Annu Rev Physiol 2017;79:517-39.

7 Kumar M, Seeger W, Voswinckel R. Senescenceassociated secretory phenotype and its possible role in chronic obstructive pulmonary disease. Am J Respir Cell Mol Biol 2014;51:323-33.

8 Pennisi E, Kaiser J, Service RF, et al. Runners-up. Science 2014;346:1444-9.

9 Loffredo FS, Steinhauser ML, Jay SM, et al. Growth differentiation factor 11 is a circulating factor that reverses age-related cardiac hypertrophy. Cell 2013;153:828-39.

10 Olson KA, Beatty AL, Heidecker B, et al. Association of growth differentiation factor $11 / 8$, putative anti-ageing factor, with cardiovascular outcomes and overall mortality in humans: analysis of the Heart and Soul and HUNT3 cohorts. Eur Heart J 2015:36:3426-34.

11 Sinha M, Jang YC, Oh J, et al. Restoring systemic GDF11 levels reverses age-related dysfunction in mouse skeletal muscle. Science 2014;344:649-52.

12 Katsimpardi L, Litterman NK, Schein PA, et al. Vascular and neurogenic rejuvenation of the aging mouse brain by young systemic factors. Science 2014;344:630-4.

13 Onodera K, Sugiura H, Yamada M, et al. Decrease in an anti-ageing factor, growth differentiation factor 11, in chronic obstructive pulmonary disease. Thorax 2017;72:893-904.

14 Campisi J. Senescent cells, tumor suppression, and organismal aging: good citizens, bad neighbors. Cell 2005; 120:513-22.

15 Beck T, Hastings RK, Gollapudi S, et al. GWAS Central: a comprehensive resource for the comparison and interrogation of genome-wide association studies. Eur J Hum Genet 2014;22:949-52.

16 Zhou Y, Jiang Z, Harris EC, et al. Circulating concentrations of growth differentiation factor 11 are heritable and correlate with Life Span. J Gerontol A Biol Sci Med Sci 2016;71:1560-3. 